\title{
A Novel Real Time Monitoring System based on WSNs and RFID for Logistics
}

\author{
Xun Wei \\ School of Economics, Southwest University for Nationalities, Si Chuan, China
}

\begin{abstract}
Currently, the monitoring system of logistics mainly depends on the transportation personnel to regularly check the status of the goods, which makes it unable to deal with accidents caused by human errors. To solve this problem, we propose a real time monitoring system based on wireless sensor networks (WSNs) and radio frequency identification (RFID) for logistics. RFID module which is beneficial to the management of goods flow information is used to identify the related information of goods. And sensor node that is attached to each goods can measure the goods behavior by a classification algorithm based on the maximum similarity and multi character recognition. The simulation shows that the proposed system can monitor the environment of the goods very well and algorithm can effectively monitor the goods behavior.
\end{abstract}

KEYWORD: logistics; wireless sensor networks; radio frequency identification; monitoring system

\section{INSTRUCTIONS}

Logistics is the management of moving goods from the point of origin to the point of consumption, including the integration of information flow, material handling, production, packaging, inventory, transportation, and warehousing. During the transporting process, unexpected accidents may occur and lead to the changes in logistics and transport environment (Chang Liu 2013). Hence, the real-time monitoring system is necessary to obtain the current condition of the goods and make the optimal decisions (Wang, X. et al. 2011). Nowadays, the monitoring system mainly relies on the transportation officers who are responsible for regularly checking status of the goods and reporting the results to the monitoring center, which makes it unable to deal with accidents caused by human error. What's more, simply relying on manpower may cause the loss of goods (Feifei Qian 2012). Hence, automatically monitoring the condition of the goods is great need of a real-time monitoring system.

The RFID (Radio Frequency Identification) technology has been widely used in the monitoring system of logistics. The canonical and interactional information stored in RFID tag is automatically collected to center system through wireless communication network. Thus, the goods can be identified and the information can be shared. Yang et al. presented the requirements of information infrastructure for humanitarian logistics centers resource management system and proposed a hybrid RFID sensor network framework (Yang H. et al. 2011). Alejandro et al. used the temperature sensor and RFID tag to track and monitor the recycled goods box (Martínez-Sala, A. S. et al. 2009). However, since RFID readers can't be deployed at anywhere, the goods can only be tracked at the given checking points, and they lack the ability to monitor the goods behavior as well (Zhi Zhang et al. 2009).

Wireless sensor networks (WSNs) are composed of many sensor nodes which communicate with each other through wireless communication. As WSNs combine distributed sensing, computation and wireless communication technologies, the conditions of the goods can be monitored on a large scale. Hence, WSNs can realize transparent monitoring of goods status during the logistics process. RuizGarcia et al. adopted the wireless sensor technology using ZigBee technology to monitor fruit storage and transport conditions (Ruiz-Garcia, L. et al. 2008). Bottero et al. proposed a traffic monitoring system based on wireless sensor networks in a logistic center. Simultaneously, a method to analyze and correct detection data was also presented to reach satisfactory accuracy even in typical installations (Bottero, M. et al. 2013).

In this paper, we design a real time monitoring system by combining RFID with WSNs for logistics. The main contributions of this paper are as follows: 1) the RFID module is used to identify the related information of goods, and it is beneficial to the management of goods flow information; 2) sensor nodes are attached to each goods and the nodes' real time positions can be obtained by GPS; and 3) an action detection algorithm based on threedimensional acceleration is proposed to monitor the goods behavior. 


\section{THE MONITORING SYSTEM STRUCTURE}

The real time monitoring system is composed of four parts: wireless sensor monitoring network, data transmission network, RFID module, and monitoring center. The structure of the system is shown in Fig.1.

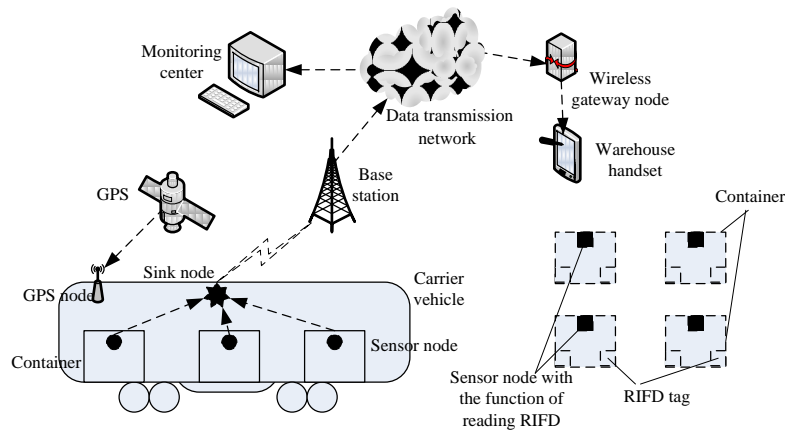

Figure 1. Structure of the real time monitoring system.

Wireless sensor monitoring network, the core of the system, is responsible for collecting the goods status data and making preliminary detection on abnormal behaviors. The sensor node is deployed inside the goods and close to the opening position. It is composed of local memory module, processor module, power supply module, three-dimensional acceleration sensor module and wireless communication module. While GPS node deployed on the top of the carrier vehicle measures the current location on a regular basis. And GPS antenna is outside the vehicle to receive the satellite signal. The Zigbee communication module, which meets IEEE802.15.4 standard, provides the geographical location information to the sensor node.

Data transmission network is composed of GSM network and its wireless communication method is GPRS. GPRS has the advantage of wide covering scope, high-speed transmission, high-quality communication, billing by flow, etc. Hence, the data transmission network can guarantee the reliability, instantaneity, stability and economics of the monitoring data transmission.

RFID module composed of electronic tag and RFID reader uses the full-duplex data communication in non-contact manner to identify the related information of goods by radio frequency. The RFID reader is a device to read the information of the tags, and it can be designed as hand-held or fixed. And the antenna is used to transmit RF signals between the tags and RFID readers.

Monitoring center consists of remote monitoring personnel, data storage device and computer. By communicating with the wireless sensor monitoring network, it can manage, coordinate and maintain the whole system

\section{DESIGN OF ALGORITHMS}

\subsection{Location Algorithm}

In the system, we use the GPS nodes to monitor the location of goods. The working principle of GPS is as follows (Feifei Qian 2012). Each satellite is equipped with the high-precision cesium atomic clock and the real-time updating database. Based on these, it continuously sends navigation data to the Earth. The receiver needs to simultaneously track four or more satellites for positioning calculation as shown in Fig. 2. In order to determine the location, the receiver must know the pseudo-distance and the location of the tracking satellites. Simultaneously, it has to consider the effect of the time deviation brought by the calculation. After the receiver has tracked and locked the satellite signal, the satellite receiver can transmit pseudo-distance measurement, two-line orbital element parameters and clock corrections parameters to the receiver. Using the two-line orbital element parameters, we can calculate the coordinates of the satellite when the satellite sends the signal. The algorithm is shown in the following equations.

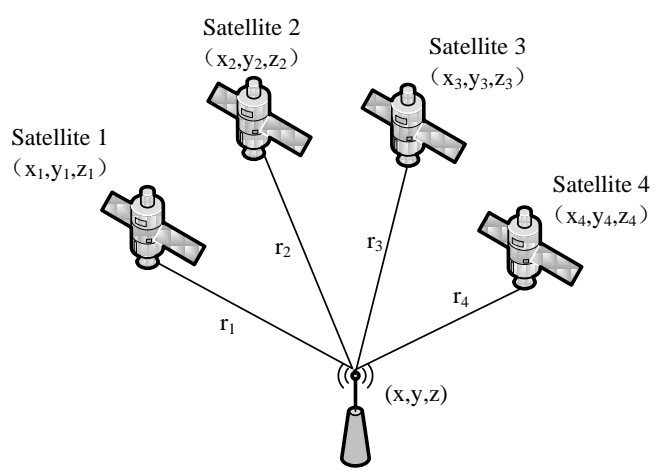

Figure 2. Localization of GPS satellites.

$$
\left\{\begin{array}{l}
r_{1}=\sqrt{\left(x_{1}-x\right)^{2}+\left(y_{1}-x\right)^{2}+\left(z_{1}-x\right)^{2}}+c t_{u} \\
r_{2}=\sqrt{\left(x_{2}-x\right)^{2}+\left(y_{2}-x\right)^{2}+\left(z_{2}-x\right)^{2}}+c t_{u} \\
r_{3}=\sqrt{\left(x_{3}-x\right)^{2}+\left(y_{3}-x\right)^{2}+\left(z_{3}-x\right)^{2}}+c t_{u} \\
r_{4}=\sqrt{\left(x_{4}-x\right)^{2}+\left(y_{4}-x\right)^{2}+\left(z_{4}-x\right)^{2}}+c t_{u}
\end{array}\right.
$$

where $(x, y, z)$ is the user's three-dimensional coordinate which is unknow; $r_{1}, r_{2}, r_{3}, r_{4}$ are respectively the distances of satellite 1 , satellite 2 , satellite 3 , satellite 4 to the anchor point, namely pseudo-distances; $c$ is the speed of light; $t_{u}$ is the clock skew of the satellites; $\left(x_{i}, y_{i}, z_{i}\right)(i=1,2,3,4)$ is the two-line orbital element parameters.

\subsection{Goods Behavior Detection Algorithm}

According to the analysis of goods behavior characteristic, we adopted a classification algorithm based on the maximum similarity and multi character recognition to determine the type of the abnormal behavior of the goods.

First, we divide the goods behavior into the following five categories.

Equilibrium: The goods are in a relatively static 
state. Usually, when the wobble of the goods is less than the threshold value during a certain period of time, we believe it is in equilibrium.

Shake: Goods swing in the horizontal direction for a long time, and show some cyclical characteristics.

Turnover: It is the process that goods fall to the ground. The flip of the goods can be considered as a plurality of continuous turnover.

Movement: Goods are promoted by a relatively stable force, and result in a change in the position within the compartment.

Strike: Goods suffer from the instantaneous collision or hitting during a short time.

Different goods behavior will make distinct damage situation to the diverse types of goods. For example, shake and turnover cause spraying and dumping of liquid goods; strike causes the combustion and explosion of the flammable and explosive objects; the human movement results in the loss of valuables. We define the shake, turnover, movement and strike as the abnormal behaviors of the goods.

Next, we make the feature analysis on the five abnormal goods behaviors. Goods behavior characteristic is the three-dimensional acceleration information of the goods under specific behavior. The sampling frequency of the used threedimensional acceleration sensor module is $150 \mathrm{~Hz}$, and it can output the acceleration of the $\mathrm{x}, \mathrm{y}, \mathrm{z}$-axes within the range of $\pm 2 g$. By placing the sensor nodes on the goods and applying different behaviors, we can collect the three-dimensional acceleration data under different behaviors. After analyzing the data, we can get three-dimensional acceleration data characteristics.

Equilibrium: The accelerations of the goods are relatively stable in three dimensions. And the maximum positive and negative offsets from the average value are both less than the threshold.

Shake: The accelerations in two dimensions are obvious bigger than the other dimension.

Turnover: The gap of the accelerations between before and after the turnover in two dimensions is bigger than the threshold.

Movement: The accelerations in three dimensions are all small, and smaller than the threshold. Moreover, the volatilities of them are all small.

Strike: The accelerations in three dimensions are all very big in the process of strike, and all bigger than the threshold. The volatilities of them are all big too.

Based on the above analysis, we can see that the different goods behavior characteristics are mainly reflected in the statistical properties of the threedimensional acceleration data (mean, variance) and the relationship among them, not only in a single characteristic. Thus, in order to distinguish the goods behaviors, we need to combine multiple statistical characteristics of the three-dimensional acceleration data.

At last, we use the multi feature recognition algorithm based on maximum similarity (MFRMS) to determine the goods behaviors. Multi feature recognition (MFR) creates a binary tree with depth of $M-1$ using the $M$ features of the three-dimensional acceleration data. All leaf nodes of the binary tree compose the entire behavior space of the goods. The route from the root to the leaf nodes denotes the decision process of the goods behaviors. The longer the route is, the more complex the goods behaviors are, and the more unobvious the goods behaviors feature is.

Maximum similarity (MS) sorts the $M$ characteristics of the data according to the priority. The higher the priority, the more obviously we determine the goods behaviors. The character with high priority is placed on the near position from the root. When the data is input, the algorithm extract and match the characteristics of the data until a certain leaf node is matched according the priority of the characteristics. Thus, the whole matching process can ensure the maximum similarity between the feature of the detection result and that of the detected data, and make sure the maximum accuracy of the goods feature.

According to the definition and characteristics analysis results of the goods behaviors, the determine situations of the feature are set as follows.

$$
\mathrm{S}_{1}: \max (\text { window })-\min (\text { window })<T 1_{r}
$$

We use the time window with the length of len and the sliding step of 1 to extract the detected data. When the gap between the maximum and minimum values of the data within the time window is smaller than $T 1_{r}$, we determined the goods state is equilibrium.

$$
\mathrm{S}_{2}:\left|\operatorname{mean}\left(a z_{0}\right)-\operatorname{mean}\left(a z_{1}\right)\right|>T 2_{r}
$$

In the equation, $a z_{0}, a z_{1}$ denote the acceleration in the $\mathrm{z}$-axis before and after the turnover. In other word, they are the two accelerations under equilibrium state before and after turnover. When the gap between the average values of the two equilibrium states in the $\mathrm{z}$-axis is bigger than the threshold $T 2_{r}$, we determined the behaviors of the goods as turnover.

$$
\mathrm{S}_{3}: \operatorname{var}\left(a_{z}(\text { start }, n)\right)>T 3_{r}
$$

where, $a_{z}($ start, $n)$ is the recorded $n$ acceleration data in the $\mathrm{z}$-axis from $t=s t a r t$ to $t=s t a r t+n$. When the variance is bigger than threshold $T 3_{r}$, we judge the behaviors of the goods as strike.

$$
\mathrm{S}_{4}: \max \left(\operatorname{var}\left(a_{x}\right), \operatorname{var}\left(a_{y}\right), \operatorname{var}\left(a_{z}\right)\right)<T 4_{r}
$$

where $\operatorname{var}\left(a_{x}\right), \operatorname{var}\left(a_{y}\right), \operatorname{var}\left(a_{z}\right)$ are the variance of the accelerations in the three dimensions respectively. When the maximum of the three is smaller than the threshold $T 4_{r}$, we determined the behaviors of the goods as movements. 


\section{EXPERIMENT RESULTS}

In order to verify the communication function of the monitoring system, we make experiments on the real time temperature and relative humidity of the goods environment. In order to conveniently test, we simplify the system. Four cartons are equipped with the sensor nodes which have the temperature and humidity sensor. The sensor nodes transmit the collected data to the computer by wireless communication. The sending data is shown in Fig. 3 and Fig. 4.

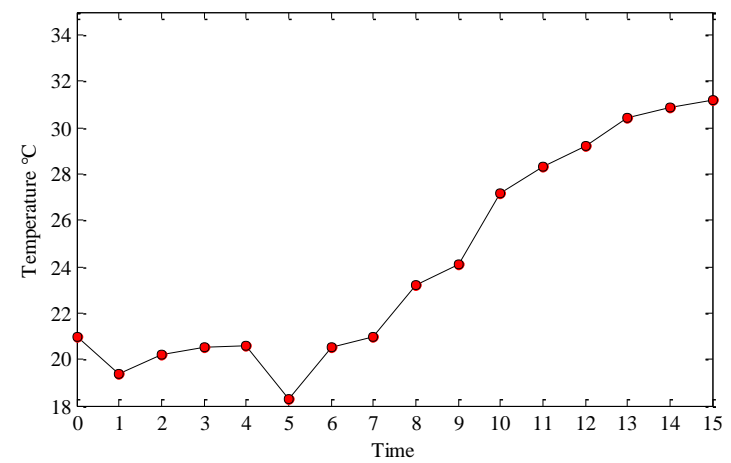

Figure 3. Temperature data of the goods environment.

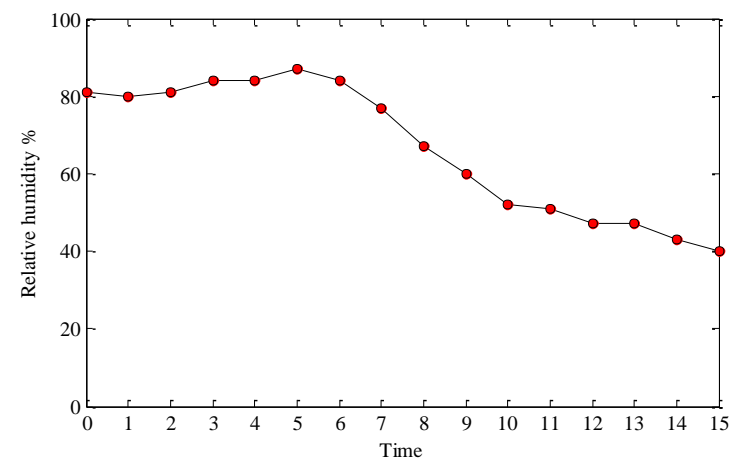

Figure 4. Relative humidity data of the goods environment.

To evaluate the function of the goods behavior detection algorithm, we detect four abnormal behaviors and the results are shown in Table 1.

Table 1. The results of abnormal behaviors

\begin{tabular}{llllc}
\hline Goods behavior & Turnover & Strike & Shake & Movement \\
\hline Accuracy rate & $100 \%$ & $100 \%$ & $100 \%$ & $96.6 \%$ \\
Missing rate & 0 & 0 & 0 & $3.4 \%$ \\
False rate & 0 & 0 & $3.5 \%$ & $3.7 \%$ \\
False result & -- & -- & $\mathrm{M}^{*}$ & Sh/E* \\
\hline
\end{tabular}

* M denotes movement, and Sh/E denotes Shake/ Equilibrium.

In Tab. 1, we can see that the algorithm can accurately detect the turnover and strike of the goods. The mainly reason is that the algorithm is based on maximum similarity and multi feature recognition, and can ensure the goods behavior with obvious characteristics detected preferentially. By contrasting between the record result and detection result of the experiments, and observing the threedimensional acceleration data signal characteristics when the behavior is detected falsely, we discover that the ambiguity of goods abnormal behavior characteristics lead to the error of the detection result. The light, stable movement of the goods is not detected and considered to be in equilibrium. Rapid acceleration starts and stops lead to a steady increase of three-dimensional acceleration, and the goods are detected falsely as movement itself. The slight shake will be detected as movement too.

\section{CONCLUSIONS}

The informatization of logistics is the inevitable trend of future development. This paper puts forward a novel real time monitoring system based on WSNs and RFID. WSNs in the real time monitor system can enable the people to conveniently and quickly obtain the behavior and location information of the goods by using sensor nodes to cooperate, process and transmit the collected data. RFID module can help manage the goods flow information by identifying the related information of goods. Through the temperature and relative humidity experiments, we can see that the proposed system can monitor the environment of the goods very well. Furthermore, the three-dimensional acceleration algorithm can effectively monitor the goods behavior as shown in the experiments.

\section{REFERENCES}

[1] Bottero, M., Dalla Chiara, B., \& Deflorio, F. P. 2013. Wireless sensor networks for traffic monitoring in a logistic centre. Transportation Research Part C: Emerging Technologies 26(3): 99-124.

[2] Chang Liu. 2013. Wireless sensor networks based active logistics status tracking system. Thesis of Huazhong University of Science and Technology.

[3] Fei fei, Qian. 2012.Research Logistic tracking information system based on wireless sensor network. Thesis of Jiangnan University.

[4] Martínez-Sala, A. S., Egea-López, E., García-Sánchez, F., \& García-Haro, J. 2009. Tracking of Returnable Packaging and Transport Units with active RFID in the grocery supply chain. Computers in Industry 60(3): 161-171.

[5] Ruiz-Garcia, L., Barreiro, P., \& Robla, J. I. 2008. Performance of ZigBee-based wireless sensor nodes for realtime monitoring of fruit logistics. Journal of Food Engineering 87(3): 405-415.

[6] Wang, X., Yuan, S., Laur, R., \& Lang, W. 2011. Dynamic localization based on spatial reasoning with RSSI in wireless sensor networks for transport logistics. Sensors and Actuators A: Physical 171(2), 421-428.

[7] Yang H., Yang L \&Yang S H. 2011. Hybrid Zigbee RFID sensor network for humanitarian logistics centre management. Journal of Network and Computer Applications 34(3): 938948.

[8] Zhi Zhang, Qiang Chen, Bergarp, T., Norman, P., Wikstrom, M., Xiaolang Yan \& Li-Rong Zheng. 2009. Wireless Sensor Networks for Logistics and Retail. International Conference on Networked Sensing Systems (INSS) Pages: 1-4. 